International Journal of Pure and Applied Mathematics

Volume 101 No. 1 2015, 9-19

ISSN: 1311-8080 (printed version); ISSN: 1314-3395 (on-line version)

url: http://www.ijpam.eu

doi: http://dx.doi.org/10.12732/ijpam.v101i1.2

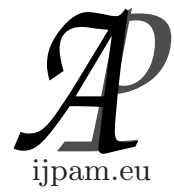

\title{
COMMON FIXED POINT THEOREMS FOR HYBRID PAIRS OF OCCASIONALLY WEAKLY COMPATIBLE MAPPINGS IN COMPLEX VALUED METRIC SPACES
}

\author{
Priyanka Nigam $^{1}$, Sandhya Shukla ${ }^{2}$ \\ ${ }^{1,2}$ Sagar Institute of Science and Technology \\ Bhopal (M.P.), INDIA
}

\begin{abstract}
In this paper we obtain some common fixed point theorems for hybrid pairs of single valued and multi-valued occasionally weakly compatible maps in complex valued metric space.
\end{abstract}

AMS Subject Classification: 47H10, 54H 25

Key Words: complex valued metric space, Common fixed point, occasionally weakly compatible mappings

\section{Introduction}

Azam et al. [3] introduced the concept of complex valued metric spaces and obtained sufficient conditions for the existence of common fixed points of a pair of contractive type mappings involving rational expressions. Subsequently many authors have studied the existence and uniqueness of the fixed points and common fixed points of self mapping in view of contrasting contractive conditions.

The study of fixed point theorems, involving four single-valued maps, began

Received: November 5, 2014

(c) 2015 Academic Publications, Ltd.

${ }^{\S}$ Correspondence author url: www.acadpubl.eu 
with the assumption that all of the maps are commuted. Sessa [12] weakened the condition of commutativity to that of pairwise weakly commuting. Jungck generalized the notion of weak commutativity to that of pairwise compatible [9] and then pairwise weakly compatible maps [10]. Jungck and Rhoades [11] introduced the concept of occasionally weakly compatible maps.

Abbas and Rhoades [2] generalized the concept of weak compatibility in the setting of single and multi-valued maps by introducing the notion of occasionally weakly compatible (owc).

In this paper we extended the result of Azam, Ahmed and Kumam [4] for hybrid pairs of occasionally weakly compatible (owc) mappings in complex valued metric space.

\section{Preliminaries}

Let $C$ be the set of complex numbers and let $z_{1}, z_{2} \in C$. Define a partial order $\leq$ on $C$ as follows: $z_{1} \leq z_{2}$ if and only if $\operatorname{Re}\left(z_{1}\right) \leq \operatorname{Re}\left(z_{2}\right), \operatorname{Im}\left(z_{1}\right) \leq \operatorname{Im}\left(z_{2}\right)$. It follows that $z_{1} \leq z_{2}$ if one of the following conditions is satisfied:

(i) $\operatorname{Re}\left(z_{1}\right)=\operatorname{Re}\left(z_{2}\right), \operatorname{Im}\left(z_{1}\right)<\operatorname{Im}\left(z_{2}\right)$,

(ii) $\operatorname{Re}\left(z_{1}\right)<\operatorname{Re}\left(z_{2}\right), \operatorname{Im}\left(z_{1}\right)=\operatorname{Im}\left(z_{2}\right)$,

(iii) $\operatorname{Re}\left(z_{1}\right)<\operatorname{Re}\left(z_{2}\right), \operatorname{Im}\left(z_{1}\right)<\operatorname{Im}\left(z_{2}\right)$,

(iv) $\operatorname{Re}\left(z_{1}\right)=\operatorname{Re}\left(z_{2}\right), \operatorname{Im}\left(z_{1}\right)=\operatorname{Im}\left(z_{2}\right)$.

In particular, we will write $z_{1} \leq z_{2}$ if one of (i), (ii) and (iii) is satisfied and we will write $z_{1}<z_{2}$ if only (iii) is satisfied.

Definition 2.1. Let $X$ be a non-empty set. Suppose that the mapping $d: X \times X \rightarrow C$ satisfies:

(i) $0 \leq d(x, y)$ for all $x, y \in X$ and $d(x, y)=0$ if and only if $x=y$;

(ii) $d(x, y)=d(y, x)$ for all $x, y \in X$;

(iii) $d(x, y) \leq d(x, z)+d(z, y)$ for all $x, y, z \in X$.

Then $d$ is called a complex valued metric on $X$ and $(X, d)$ is called a complex valued metric space.

Definition 2.2. [4] Let $(X, d)$ be a complex-valued metric space. 
We denote the family of nonempty, closed and bounded subsets of a complex valued metric space by $C B(X)$.

From now on, we denote $s\left(z_{1}\right)=\left\{z_{2} \in C: z_{1} \leq z_{2}\right\}$ for $z_{1} \in C$, and $s(a, B)=\cup_{b \in B} s(d(a, b))=\cup_{b \in B}\{z \in C: d(a, b) \leq z\}$ for $a \in X$ and $B \in$ $C B(X)$.

For $A, B \in C B(X)$, we denote

$$
s(A, B)=\left(\cap_{a \in A} s(a, B)\right) \cap\left(\cap_{b \in B} s(b, A)\right) .
$$

Remark 2.3. [4] Let $(X, d)$ be a complex-valued metric space. If $C=$ $R$, then $(X, d)$ is a metric space. Moreover, for $A, B \in C B(X), H(A, B)=$ inf $s(A, B)$ is the Hausdorff distance induced by $d$.

Definition 2.4. A point $x \in X$ is called a coincidence point (resp. fixed point) of $A: X \rightarrow X, B: X \rightarrow C B(X)$ if $A x \in B x$ (resp. $x=A x \in B x$ ).

Definition 2.5. Maps $f: X \rightarrow X$ and $T: X \rightarrow C B(X)$ are said to be weakly compatible if they commute at their coincidence points, that is $f x \in T x$ for some $x \in X$ then $f T x=T f x$.

Definition 2.6. Maps $f: X \rightarrow X$ and $T: X \rightarrow C B(X)$ are said to be occasionally weakly compatible (owc) if and only if there exist some point $x$ in $X$ such that $f x \in T x$ and $f T x \subseteq T f x$.

For example, if we take $X=[0, \infty)$ with usual metric then $f: X \rightarrow X$ and $T: X \rightarrow C B(X)$ defined by $f(x)=x^{2}$ and $T(x)=\left[0, \frac{1}{x}\right]$ are occasionally weakly compatible.

Example 2.7. let $X=[0, \infty)$ with usual metric. Define $f: X \rightarrow X, T$ : $X \rightarrow C B(X)$ by

$$
f x= \begin{cases}0, & 0 \leq x<1 \\ x+1, & 1 \leq x<\infty\end{cases}
$$

and

$$
T x= \begin{cases}\{x\}, & 0 \leq x<1 \\ {[1, x+2],} & 1 \leq x<\infty\end{cases}
$$

It, can be easily verified that $x=1$, is coincidence point of $f$ and $T$, but $f$ and $T$ are not weakly compatible there. However the pair $\{f, T\}$ is occasionally weakly compatible, since the pair $\{f, T\}$ is weakly compatible at $x=0$. 


\section{Main Results}

Theorem 3.1. Let $(X, d)$ be a Complex valued metric space $f, g: X \rightarrow X$ and $F, G: X \rightarrow C B(X)$ be single valued and multi-valued maps respectively such that the pairs $\{f, F\}$ and $\{g, G\}$ are owc and satisfy inequality

$$
\alpha d(f x, g y)+\beta d(f x, G y)+\gamma d(g y, F x) \in s(F x, G y)
$$

for all $x, y \in X$ for which $f x \neq g y$, where $\alpha, \beta, \gamma>0$ and $(\alpha+\beta+\gamma)<1$. Then $f, g, F$ and $G$ have a unique common fixed point.

Proof. Since the pairs $\{f, F\}$ and $\{g, G\}$ are owc, then there exists two points $x, y \in X$ such that $f x \in F x, f F x \subseteq F f x$ and $g y \in G y, g G y \subseteq G g y$.

First we prove that $f x=g y$. If not then by (3.1) we get

$$
\alpha d(f x, g y)+\beta d(f x, G y)+\gamma d(g y, F x) \in s(F x, G y)
$$

This implies that

$$
\begin{aligned}
& \alpha d(f x, g y)+\beta d(f x, G y)+\gamma d(g y, F x) \in\left(\bigcap_{f x \in F x} s(F x, G y)\right) \\
& \alpha d(f x, g y)+\beta d(f x, G y)+\gamma d(g y, F x) \in s(F x, G y)=\bigcup_{g y \in G y} s(d(f x, g y)) \\
& \alpha d(f x, g y)+\beta d(f x, G y)+\gamma d(g y, F x) \in s(d(f x, g y))
\end{aligned}
$$

That is,

$$
d(f x, g y) \leq \alpha d(f x, g y)+\beta d(f x, G y)+\gamma d(g y, F x)
$$

Since $f x \in F x$ and $g y \in G y$, so we have

$$
\begin{aligned}
& d(F x, G y) \leq \alpha d(F x, G y)+\beta d(F x, G y)+\gamma d(G y, F x) \\
& \text { or } \quad d(F x, G y) \leq(\alpha+\beta+\gamma) d(F x, G y) \\
& \text { as } \quad(\alpha+\beta+\gamma)<1, \text { this implies that } \\
& d(F x, G y)<d(F x, G y),
\end{aligned}
$$

a contradiction, and hence $f x=g y$.

Next we claim that $x=f x$. If not then by (3.1) we get

$$
\alpha d(f x, g f x)+\beta d(f x, G f x)+\gamma d(g f x, F x) \in s(F x, G f x)
$$


This implies that

$$
\begin{aligned}
& \alpha d(f x, g f x)+\beta d(f x, G f x)+\gamma d(g f x, F x) \in\left(\bigcap_{f x \in F x} s(f x, G f x)\right) \\
& \alpha d(f x, g f x)+\beta d(f x, G f x)+\gamma d(g f x, F x) \in s(f x, G f x) \\
& =\bigcup_{g x \in G x} s(d(f x, g f x)) \\
& \text { or } \alpha d(f x, g f x)+\beta d(f x, G f x)+\gamma d(g f x, F x) \in s(d(f x, g f x))
\end{aligned}
$$

That is,

$$
d(f x, g f x) \leq \alpha d(f x, g f x)+\beta d(f x, G f x)+\gamma d(g f x, F x)
$$

Since $f x \in F x$ and $g y \in G y$, and $\{f, F\}$ and $\{g, G\}$ are owc so we have,

$$
\begin{aligned}
d(F x, G f x) & \leq \alpha d(F x, G f x)+\beta d(F x, G f x)+\gamma d(G f x, F x) \\
\text { or } \quad d(F x, G f x) & \leq(\alpha+\beta+\gamma) d(F x, G f x) \\
\text { as } \quad(\alpha+\beta+\gamma) & <1, \text { this implies that } \\
d(F x, G f x) & <d(F x, G f x),
\end{aligned}
$$

which is again a contradiction and the claim follows. Similarly we obtain $y=$ $g y$. Thus $f, g, F$ and $G$ have a common fixed point. Uniqueness follows from (3.1).

Theorem 3.2. Let $(X, d)$ be a Complex valued metric space $f, g: X \rightarrow X$ and $F, G: X \rightarrow C B(X)$ be single valued and multi-valued maps respectively such that the pairs $\{f, F\}$ and $\{g, G\}$ are owc and satisfy inequality

$$
\begin{aligned}
& k \max \{d(f x, g y), d(f x, F x), d(f x, G y), d(g y, G y), d(g y, F x)\} \\
& \in s(F x, G y)
\end{aligned}
$$

for all $x, y \in X$ for which $f x \neq g y$ and $0<k<1$. Then $f, g, F$ and $G$ have a unique common fixed.

Proof. Since the pairs $\{f, F\}$ and $\{g, G\}$ are owc, then there exists two elements $x, y \in X$ such that $f x \in F x, f F x \subseteq F f x$ and $g y \in G y, g G y \subseteq G g y$.

First we prove that $f x=g y$. If not then by (3.2) we get

$$
k \max \{d(f x, g y), d(f x, F x), d(f x, G y), d(g y, G y), d(g y, F x)\} \in s(F x, G y)
$$


This implies that

$$
\begin{aligned}
& k \max \{d(f x, g y), d(f x, F x), d(f x, G y), d(g y, G y), d(g y, F x)\} \\
& \in\left(\bigcap_{f x \in F x} s(f x, G y)\right) \\
& k \max \{d(f x, g y), d(f x, F x), d(f x, G y), d(g y, G y), d(g y, F x)\} \\
& \in s(f x, G y)=\bigcup_{g y \in G y} s(d(f x, g y)) \\
& k \max \{d(f x, g y), d(f x, F x), d(f x, G y), d(g y, G y), d(g y, F x)\} \in s(d(f x, g y))
\end{aligned}
$$

That is,

$$
d(f x, g y) \leq k \max \{d(f x, g y), d(f x, F x), d(f x, G y), d(g y, G y), d(g y, F x)\}
$$

Since $f x \in F x$ and $g y \in G y$, so we have

$$
\begin{aligned}
d(F x, G y) & \leq k \max \{d(F x, G y), d(F x, F x), d(F x, G y), d(G y, G y), d(G y, F x)\} \\
& \leq k d(F x, G y)
\end{aligned}
$$

as $0<k<1$, this implies that

or $d(F x, G y)<d(F x, G y)$,

a contradiction, and hence $f x=g y$.

Next we claim that $x=f x$. If not then by (3.2) we get

$$
\begin{array}{r}
k \max \{d(f x, g f x), d(f x, F x), d(f x, G f x), d(g f x, G f x), d(g f x, F x)\} \\
\in s(F x, G f x)
\end{array}
$$

This implies that

$$
\begin{aligned}
& k \max \{d(f x, g f x), d(f x, F x), d(f x, G f x), d(g f x, G f x), d(g f x, F x)\} \\
& \in s(f x, G f x) \\
& \text { or } \\
& k \max \{d(f x, g f x), d(f x, F x), d(f x, G f x), d(g f x, G f x), d(g f x, F x)\} \\
& =\bigcup_{g x \in G x} s(d(f x, g f x)) \\
& \text { or } \\
& k \max \{d(f x, g f x), d(f x, F x), d(f x, G f x), d(g f x, G f x), d(g f x, F x)\}
\end{aligned}
$$




$$
\in s(d(f x, g f x))
$$

That is

$$
\begin{aligned}
& d(f x, g f x) \\
& \quad \leq k \max \{d(f x, g f x), d(f x, F x), d(f x, G f x), d(g f x, G f x), d(g f x, F x)\} .
\end{aligned}
$$

Since $f x \in F x$ and $g y \in G y$ and $\{f, F\}$ and $\{g, G\}$ are owc so we have,

$$
\begin{aligned}
d(F x, G f x) \leq & k \max \{d(F x, G f x), d(f x, F x), d(F x, G f x), \\
& d(g f x, G f x), d(G f x, F x)\} \\
\leq & k d(F x, G f x)
\end{aligned}
$$

as $0<k<1$, this implies that or $d(F x, G f x)<d(F x, G f x)$,

which is again a contradiction and the claim follows. Similarly we obtain $y=g y$. Thus $f, g, F$ and $G$ have a common fixed point. Uniqueness follows from (3.2).

Theorem 3.3. Let $(X, d)$ be a Complex valued metric space $f, g: X \rightarrow X$ and $F, G: X \rightarrow C B(X)$ be single valued and multi-valued maps respectively such that the pairs $\{f, F\}$ and $\{g, G\}$ are owc and satisfy inequality

$$
\begin{aligned}
k \max \left\{d(f x, g y), d(f x, F x), d(g y, G y), \frac{d(f x, G y)+d(g y, F x)}{2}\right\} & \\
\in & s(F x, G y)
\end{aligned}
$$

for all $x, y \in X$ for which $f x \neq g y$ and $0<k<1$. Then $f, g, F$ and $G$ have a unique common fixed.

Proof. Clearly the result follows from Theorem 3.2.

Theorem 3.4. Let $(X, d)$ be a Complex valued metric space $f, g: X \rightarrow X$ and $F, G: X \rightarrow C B(X)$ be single valued and multi-valued maps respectively such that the pairs $\{f, F\}$ and $\{g, G\}$ are owc and satisfy inequality

$$
\begin{aligned}
& k \max \left\{d(f x, g y), \frac{d(f x, F x)+d(g y, G y)}{2}, \frac{d(f x, G y)+d(g y, F x)}{2}\right\} \\
& \in s(F x, G y)
\end{aligned}
$$

for all $x, y \in X$ for which $f x \neq g y$ and $0<k<1$. Then $f, g, F$ and $G$ have a unique common fixed. 
Proof. Clearly the result follows from Theorem 3.2.

Theorem 3.5. Let $(X, d)$ be a Complex valued metric space $f, g: X \rightarrow X$ and $F, G: X \rightarrow C B(X)$ be single valued and multi-valued maps respectively such that the pairs $\{f, F\}$ and $\{g, G\}$ are owc and satisfy inequality

$$
k \max \left\{d(f x, g y),\left(1-\frac{d(g y, G y)+d(f x, G y)}{d(f x, F x)+d(g y, F x)}\right)\right\} \in s(F x, G y)
$$

for all $x, y \in X$ for which $f x \neq g y$ and $0<k<1$. Then $f, g, F$ and $G$ have a unique common fixed.

Proof. Since the pairs $\{f, F\}$ and $\{g, G\}$ are owc, then there exists two points $x, y \in X$ such that $f x \in F x, f F x \in F f x$ and $g y \in G y, g G y \subseteq G g y$.

First we prove that $f x=g y$. If not then by (3.5) we get

$$
k \max \left\{d(f x, g y),\left(1-\frac{d(g y, G y)+d(f x, G y)}{d(f x, F x)+d(g y, F x)}\right)\right\} \in s(F x, G y)
$$

This implies that

$$
\begin{aligned}
& k \max \left\{d(f x, g y),\left(1-\frac{d(g y, G y)+d(f x, G y)}{d(f x, F x)+d(g y, F x)}\right\} \in\left(\bigcap_{f x \in F x} s(f x, G y)\right)\right. \\
& k \max \left\{d(f x, g y),\left(1-\frac{d(g y, G y)+d(f x, G y)}{d(f x, F x)+d(g y, F x)}\right\} \in s(f x, G y)\right. \\
& =\left(\bigcup_{g y \in G y} s(d(f x, G y))\right) \\
& k \max \left\{d(f x, g y),\left(1-\frac{d(g y, G y)+d(f x, G y)}{d(f x, F x)+d(g y, F x)}\right)\right\} \in s(d(f x, g y))
\end{aligned}
$$

That is,

$$
d(f x, g y) \leq k \max \left\{d(f x, g y),\left(1-\frac{d(g y, G y)+d(f x, G y)}{d(f x, F x)+d(g y, F x)}\right)\right\}
$$

Since $f x \in F x$ and $g y \in G y$, so we have

$$
d(F x, G y) \leq k \max \left\{d(F x, G y),\left(1-\frac{d(G y, G y)+d(F x, G y)}{d(f x, F x)+d(G y, F x)}\right)\right\}
$$




$$
\begin{aligned}
& =k \max \left\{d(F x, G y),\left(1-\frac{d(F x, G y)}{d(G y, F x)}\right)\right\} \\
& =k \max \{d(F x, G y), 0\} \\
& \leq k d(F x, G y)
\end{aligned}
$$

as $0<k<1$, this implies that

$$
d(F x, G y)<d(F x, G y),
$$

a contradiction, and hence $f x=g y$.

Next we claim that $x=f x$. If not then by (3.5) we get

$$
k \max \left\{d(f x, g f x),\left(1-\frac{d(g f x, G f x)+d(f x, G f x)}{d(f x, F x)+d(g f x, F x)}\right)\right\} \in s(F x, G f x)
$$

This implies that,

$$
\begin{aligned}
& k \max \left\{d(f x, g f x),\left(1-\frac{d(g f x, G f x)+d(f x, G f x)}{d(f x, F x)+d(g f x, F x)}\right)\right\} \in\left(\bigcap_{f x \in F x} s(f x, G f x)\right) \\
& k \max \left\{d(f x, g f x),\left(1-\frac{d(g f x, G f x)+d(f x, G f x)}{d(f x, F x)+d(g f x, F x)}\right)\right\} \in s(f x, G f x) \\
& =\left(\bigcup_{g x \in G x} s(d(f x, g f x))\right) \\
& k \max \left\{d(f x, g f x),\left(1-\frac{d(g f x, G f x)+d(f x, G f x)}{d(f x, F x)+d(g f x, F x)}\right)\right\} \in s(d(f x, g f x))
\end{aligned}
$$

That is,

$$
d(f x, g f x) \leq k \max \left\{d(f x, g f x),\left(1-\frac{d(g f x, G f x)+d(f x, G f x)}{d(f x, F x)+d(g f x, F x)}\right)\right\}
$$

Since $f x \in F x$ and $g y \in G y$ and $\{f, F\}$ and $\{g, G\}$ so we have,

$$
\begin{aligned}
d(F x, G f x) & \leq k \max \left\{d(F x, G f x),\left(1-\frac{d(G f x, G f x)+d(F x, G f x)}{d(f x, F x)+d(G f x, F x)}\right)\right\} \\
& =k \max \left\{d(F x, G f x),\left(1-\frac{d(F x, G f x)}{d(G f x, F x)}\right)\right\}
\end{aligned}
$$




$$
\begin{aligned}
& =k \max \{d(F x, G f x), 0\} \\
& \leq k d(F x, G f x)
\end{aligned}
$$

as $0<k<1$, this implies that

$$
d(F x, G f x)<d(F x, G f x)
$$

which is again a contradiction and the claim follows. Similarly we obtain $y=$ $g y$. Thus $f, g, F$ and $G$ have a common fixed point. Uniqueness follows from (3.5).

Theorem 3.6. Let $(X, d)$ be a Complex valued metric space $f, g: X \rightarrow X$ and $F, G: X \rightarrow C B(X)$ be single valued and multi-valued maps respectively such that the pairs $\{f, F\}$ and $\{g, G\}$ are owc and satisfy inequality

$$
\begin{array}{r}
\alpha d(f x, g y)^{p}+(1-\alpha) \max \left\{(d(f x, G y))^{p},(d(g y, F x))^{p},(d(f x, F x))^{p}\right. \\
\left.(d(g y, G y))^{p}\right\} \in s(F x, G y)^{p}
\end{array}
$$

for all $x, y \in X$ for which $f x \neq g y, p \geq 1$ and $\alpha \in(0,1]$. Then $f, g, F$ and $G$ have a unique common fixed.

Proof. Clearly the result follows from Theorem 3.1.

\section{References}

[1] M. Aamri and D.El Moutawakil, Some new common fixed point theorems under strict contractive conditions, J. Math. Anal. Appl., 270 (2002), 181188.

[2] M. Abbas and B.E. Rhoades, Common fixed point theorems for hybrid pairs of occasionally weakly compatible mappings satisfying generalized contractive condition of integral type, Fixed Point Theory Appl., 2007, Art. ID 54101, 9pp. (2008i:540).

[3] A. Azam, B. Fisher, and M. Khan, Common fixed point theorems in complex valued metric spaces, Numerical Functional Analysis and Optimization, Vol. 32, no. 3, pp. 243-253, 2011. 
[4] A. Azam , J. Ahmed and P. Kumam, Common fixed point theorems for multi-valued mappings in complex-valued metric spaces, Journal of Inequalities $\&$ Applications, pp. 2-12, 013:578.

[5] S. Bhatt, S. Chaukiyal, and R. C. Dimri, A common fixed point theorem for weakly compatible maps in complex valued metric spaces, International Journal of Mathematical Sciences \& Applications, Vol. 1, no. 3, pp. 13851389, 2011.

[6] S. Chandok and D. Kumar, Some common Fixed point results for rational type contraction mappings in complex valued metric spaces, Journal of operators, Vol. 2013 , Article ID813707,6 pages, 2013.

[7] S. Chauhan, W. Sintunavarat, and P. Kumam, Common fixed point theorems for weakly compatible mappings in fuzzy metric spaces using (JCLR)property, Applied Mathematics, Vol. 3, no. 9, pp. 976-982, 2012.

[8] Xin-Qi Hu, Common Coupled Fixed Point Theorems for Contractive Mappings in Fuzzy Metric Spaces, Fixed Point Theory and Applications, Vol.2011, article id 363716,14 pages.

[9] G.Jungck, Compatible mappings and common fixed points, International Journal of Mathematics and Mathematical Sciences, Vol. 9, No. 4, 1986, 771-779. (87m:54122)

[10] G.Jungck, Common fixed points for noncontinuous nonself maps on nonmetric spaces, Far East Journal of Mathematical Sciences, Vol. 4, No. 2, 1996, 199-215.

[11] G.Jungck and B. E. Rhoades, Fixed point theorems for occasionally weakly compatible mappings, Fixed Point Theory, Vol. 7, no. 2, pp. 287-296, 2006.

[12] S.Sessa, On a weak commutativity condition of mappings in fixed point considerations, Publications de l' Institute Mathe'matique, Vol. 32, No. 46, 1982, 149-153. (85f:54107)

[13] W. Sintunavarat and P. Kumam, Generalized common fixed point theorems in complex valued metric spaces and applications, Journal of Inequalities and Applications, Vol. 2012, article 84, 2012.

[14] R. K. Verma and H. K. Pathak, Common fixed point theorems using property (E.A) in complex-vauled metric spaces, Thai Journal of Mathematics. In press. 
